\title{
Radii of halo nuclei from cross section measurements
}

\author{
J. S. Al-Khalili, J. A. Tostevin, and I. J. Thompson \\ Department of Physics, University of Surrey, Guildford, Surrey, GU2 5XH United Kingdom
}

(Received 10 April 1996)

\begin{abstract}
The root mean square matter radii of halo nuclei provide a basic measure in constructing, constraining, and assessing theoretical models of halo structures. We consider corrections to static density (optical limit) Glauber model calculations of reaction cross sections of such nuclei at high energy giving careful consideration to their intrinsic few-body structure and the adiabatic nature of the halo nucleus-target interaction. We take as important examples the loosely bound two- and three-body systems ${ }^{11} \mathrm{Be},{ }^{6} \mathrm{He},{ }^{11} \mathrm{Li}$, and ${ }^{14} \mathrm{Be}$. The contribution of the valence particles to the calculated reaction cross sections are shown to be significantly reduced, requiring increased halo radii to reproduce experimental data. The implications of these changes for structure models of extended two- and three-body systems are discussed. [S0556-2813(96)04810-8]

PACS number(s): 21.10.Gv, 11.80.Fv, 25.10. $+\mathrm{s}, 27.20 .+\mathrm{n}$
\end{abstract}

\section{INTRODUCTION}

Interaction cross section measurements at high energies have been used to estimate the extent of matter densities of exotic nuclei produced in high energy fragmentation reactions [1-3]. The accuracy of the experimental cross section data involved is in many cases extremely impressive holding out the possibility of placing quite stringent limits upon these nuclear sizes. At secondary beam energies of several hundred $\mathrm{MeV}$ per nucleon the static density [4] or optical limit of the Glauber model [5-7] has been the basis for determining empirical radii. Analyses of data for a range of projectiles on a ${ }^{12} \mathrm{C}$ target have shown deduced radii to be quite insensitive to the precise radial shapes of the density distributions assumed $[3,8]$.

This static density model neglects correlations between the projectile (and target) constituents [6] and has been found to work well for spatially localized nuclei [9]. For weakly bound systems however the intrinsic few-body character, or granularity, of the projectiles imply strong spatial correlations between the constituents. For such systems reaction models $[5,10,11]$ make an adiabatic approximation, freezing the position coordinates of the few-body projectile constituents during the interaction. Physical observables are then obtained by suitably averaging the resulting position dependent reaction amplitudes over the relevant position probability distributions of these constituents. This approach forms the basis of the calculations of the present paper. A shorter discussion of these ideas has been presented elsewhere [4].

Consider a three-body projectile comprising a pair of neutrons weakly bound to a more massive core. For an impact parameter $b$ of the projectile, Fig. 1, at which its density (shaded circle) overlaps the target, many configurations of the three constituent bodies will not in fact overlap the target. The cross section calculation therefore requires a summation over all possible configurations of the three bodies with each weighted according to the best available few-body descriptions of the systems involved. The expectation is that the contribution to the cross section from large impact parameters will be reduced. Support for this expectation comes from the work of Nishioka and Johnson [12] who investi- gated related few-body effects for light-ion composite projectiles.

The accuracy of static density calculations of reaction cross sections for ${ }^{11} \mathrm{Li}$ was considered also by Takigawa et al. [13] using a simplified two-body model of the halo density. That work demonstrated clearly the convergence of two-body and static density descriptions in the limit of tight valence nucleon binding, and that the two-body correlations lead to a reduction in the calculated reaction cross sections. The model failed however to account for the very special three-body correlations present in ${ }^{11} \mathrm{Li}$ and similar Borromean [14] nuclei, with no bound binary subsystems. The overestimation of cross sections in the static density model is also commented upon by Chulkov et al. [8] and discussed by Ogawa et al. [15].

We examine quantitatively the implications of such a fewbody description upon the deduced matter radii of halo nuclei. We concentrate on the one- and two-neutron halo nuclei ${ }^{11} \mathrm{Be},{ }^{6} \mathrm{He},{ }^{11} \mathrm{Li}$, and ${ }^{14} \mathrm{Be}$. Cross section data are available for each of these composite nuclei and for their associated core subsystems $\left({ }^{10} \mathrm{Be},{ }^{4} \mathrm{He},{ }^{9} \mathrm{Li}\right.$, and $\left.{ }^{12} \mathrm{Be}\right)$ on a ${ }^{12} \mathrm{C}$ target at energies of order $800 \mathrm{MeV} /$ nucleon [1,3,16], as are data for the nucleon- ${ }^{12} \mathrm{C}$ system [17]. Having considered the results of calculations at $800 \mathrm{MeV} /$ nucleon we will also apply the model to the ${ }^{11} \mathrm{Li}$ system at $400 \mathrm{MeV} /$ nucleon where

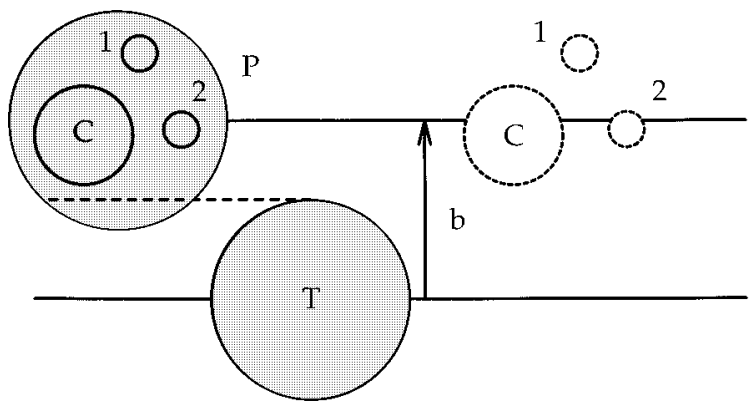

FIG. 1. Schematic representation of the static density (shaded circle) and few-body (frozen coordinate) treatments of the threebody projectile- $(P)$ target $(T)$ collision at impact parameter $b$. In the spatial configuration drawn the few-body projectile does not overlap the target. 
there are experimental data for the composite nucleus but not for the core. A key ingredient in our description is to use the static density approximation to each projectile constituenttarget subsystem. Additionally, the adiabatic (frozen coordinate) treatment of these constituents allows us to study carefully the implications for calculated cross sections of a realistic treatment of the two- and three-body nature of the projectile structures through the use of realistic few-body wave functions.

\section{FORMALISM}

In the limit of large orbital angular momenta, the reaction cross section for a projectile $P$ incident upon a target $T$ can be written [5]

$\sigma_{R}(P)=2 \pi \int_{0}^{\infty} d b b\left[1-T_{P}(b)\right]=2 \pi \int_{0}^{\infty} d b b\left[1-\left|S_{P}^{\mathrm{el}}(b)\right|^{2}\right]$,

where $T_{P}(b)$, the squared modulus of the elastic $S$ matrix for the projectile-target system, is the projectile transparency. $T_{P}(b)$ determines the probability, at impact parameter $b$ of the projectile center of mass, that the projectile survives the collision to emerge in the elastic channel. At incident energies such that Glauber theory [5] is appropriate, the calculation of this elastic $S$ matrix is particularly elegant. For instance, if the projectile-target effective interaction (optical potential) $V_{P}$ were known then

$$
\begin{gathered}
S_{P}^{\mathrm{el}}(b)=\exp \left[i \mathcal{X}_{P}(b)\right] \\
\mathcal{X}_{P}(b)=-\frac{1}{\hbar v} \int_{-\infty}^{\infty} d z V_{P}\left(\sqrt{b^{2}+z^{2}}\right)
\end{gathered}
$$

with $v$ the relative velocity of the two nuclei.

Of particular interest is the case when the projectile nucleus is a composite of mass $A_{P}$. In addition, for halo nuclei, we are dealing with systems with structures which have a more natural physical description in terms of a strongly correlated $n$-body system. Here $n$, the number of constituents, which can be individual nucleons or clusters of nucleons, is usually less than $A_{P}$ and depends upon the projectile involved and the model assumed. Two-body $(n=2)$ and three-body $(n=3)$ models are commonly used and each body is assumed to interact with the target through a twobody interaction. In all cases, for a composite projectile, Glauber theory first freezes the constituent particle positions: the adiabatic approximation. Each constituent $j$ is then assumed to follow its individual straight line path at impact parameter $b_{j}$ (Fig. 2) through the interaction region with the target. The projectile elastic $S$ matrix, entering Eq. (1), is then computed by projecting and/or averaging these different component-target amplitudes over the projectile ground state $\left|\Phi_{0}^{(n)}\right\rangle$. Thus, for a given $n$-body projectile and wave function, we derive the elastic amplitudes

$$
S_{P}^{\mathrm{el}(n)}(b)=\left\langle\Phi_{0}^{(n)}\left|\mathcal{S}^{(n)}\right| \Phi_{0}^{(n)}\right\rangle .
$$

Since the interaction operator of the constituents $j$ with the target is the sum of two-body interactions, the $S$-matrix operator $\mathcal{S}^{(n)}$ can be written

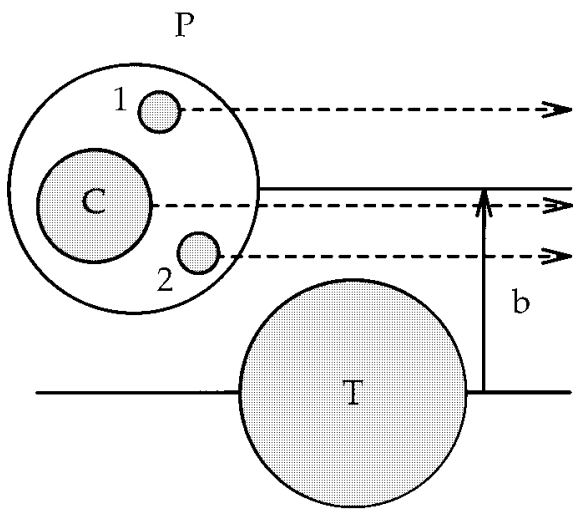

FIG. 2. Representation of the few-body Glauber (adiabatic) treatment of a three-body projectile- $(P)$ target $(T)$ collision at center of mass impact parameter $b$, showing the assumed straight line trajectories of each constituent at its own impact parameter.

$$
\mathcal{S}^{(n)}=\exp \left[i \sum_{j=1}^{n} \mathcal{X}_{j}\left(b_{j}\right)\right]=\prod_{j=1}^{n} S_{j}\left(b_{j}\right) .
$$

Here $\left|\Phi_{0}^{(n)}\right\rangle$ is the state of relative motion of the $n$ constituents in the projectile and the bra-ket denotes integration over these relative coordinates. In the present work we are particularly interested in systems comprising a heavy localized core of $A_{\mathrm{C}}$ nucleons and $(n-1)$ loosely bound valence nucleons. For those of the $n$ bodies which are nucleons, the $S_{j}\left(b_{j}\right)$ are the Glauber $S$ matrices for the nucleon-target system, subsequently denoted $S_{N}\left(b_{j}\right)$, and should be consistent with independent empirical data for the nucleon-target system. If these interact via a potential $V_{N}$, then of course, for $j=1, \ldots, n-1$,

$$
\begin{gathered}
S_{j}\left(b_{j}\right) \equiv S_{N}\left(b_{j}\right)=\exp \left[i \mathcal{X}_{N}\left(b_{j}\right)\right], \\
\mathcal{X}_{N}\left(b_{j}\right)=-\frac{1}{\hbar v} \int_{-\infty}^{\infty} d z_{j} V_{N}\left(\sqrt{b_{j}^{2}+z_{j}^{2}}\right) .
\end{gathered}
$$

When $j=C$, a composite core cluster with internal state $\left|\phi_{C}\right\rangle$, the Glauber core-target $S$ matrix is, by analogy with Eqs. (3) and (4),

$$
S_{j}\left(b_{j}\right) \equiv S_{C}\left(b_{C}\right)=\left\langle\phi_{C}|\mathcal{S}| \phi_{C}\right\rangle,
$$

where $\mathcal{S}$ is the $S$-matrix operator for the core constituents. To the extent therefore that the core state $\left|\phi_{C}\right\rangle$ in the halo nucleus is reliably represented by the ground state of the free core nucleus, $S_{C}\left(b_{C}\right)$ will describe free core-target elastic scattering and should also be consistent with experimental data for that system. In the case of such an $n$-body [core + $(n-1)$ nucleon] projectile therefore

$$
S_{P}^{\mathrm{el}(n)}(b)=\left\langle\Phi_{0}^{(n)}\left|S_{C}\left(b_{C}\right) \prod_{j=1}^{(n-1)} S_{N}\left(b_{j}\right)\right| \Phi_{0}^{(n)}\right\rangle .
$$

The explicit treatment of the composite nature of the target nucleus, of mass $A_{T}$, can of course be carried out in a perfectly symmetric manner with obvious generalization of the notation. 
This few-body projectile description is not the philosophy behind the static density limit, which is derived on the basis of the combined $A_{P}+A_{T}$ nucleon system and an approximate description of the pairwise effective $N N$ amplitudes. The derivation of the static density approximation to the nucleus-nucleus elastic $S$ matrix, and hence the projectile transparency, is formulated from a number of different viewpoints in the literature $[5,6,18]$ and will not be reproduced here. The simplest version, in the context of Eq. (2), is the use of a " $t \rho \rho$ " single scattering approximation [18] to relate the nucleus-nucleus optical potential to the target and projectile ground state densities; that is retaining the leading order term in the cumulant expansion of the multiple scattering series [19]. The resulting elastic $S$ matrix, having made zerorange and forward scattering approximations to the $N N$ amplitude, and then retained only its imaginary part, is

$$
S_{P}^{\mathrm{SD}}(b)=\exp \left[-\frac{\vec{\sigma}_{N N}^{P T}}{2} \int d^{2} \vec{x} \rho_{P}^{(z)}(|\vec{x}|) \rho_{T}^{(z)}(|\vec{b}-\vec{x}|)\right],
$$

where $\bar{\sigma}_{N N}^{P T}$ is the isospin weighted nucleon-nucleon cross section appropriate for the specific projectile and target, evaluated at the appropriate $N N$ relative energy. The ground state matter distributions $\rho_{P}$ and $\rho_{T}$ of the projectile and target appear in Eq. (8) as $z$-integrated densities,

$$
\rho_{i}^{(z)}(b)=\int_{-\infty}^{\infty} d z \rho_{i}\left(\sqrt{b^{2}+z^{2}}\right)
$$

or thickness functions. The crucial aspect of this static density limit is that only the projectile ground state densities enter the calculation and few-body correlations (the granular nature of the projectiles) does not enter explicitly. Equation (8), when substituted in Eq. (1), generates the static density approximation to the reaction cross section $\sigma_{R}^{\mathrm{SD}}(P)$ which has formed the basis of previous analyses of experimental cross section data and of deduced halo and core nucleus matter radii. The model has been shown to work well when the projectile and target are regular nuclei, in the sense that they are spatially localized and all nucleons occupy a well-defined mean field volume [9].

When considering halo nuclei, it is the constituent coretarget and valence particle-target two-body systems which have this localized nature. It is expected therefore that these binary systems can be treated reliably in the static density limit. Thus, to describe the core-target system we will use the static density elastic $S$ matrix, analogous to Eq. (8),

$$
S_{C}^{\mathrm{SD}}\left(b_{C}\right)=\exp \left[-\frac{\bar{\sigma}_{N N}^{C T}}{2} \int d^{2} \vec{x} \rho_{C}^{(z)}(|\vec{x}|) \rho_{T}^{(z)}\left(\left|\vec{b}_{C}-\vec{x}\right|\right)\right],
$$

with $\rho_{C}$ the density of the core. Similarly, for each valence nucleon $(N)$,

$$
S_{N}^{\mathrm{SD}}\left(b_{j}\right)=\exp \left[-\frac{\vec{\sigma}_{N N}^{N T}}{2} \rho_{T}^{(z)}\left(b_{j}\right)\right]
$$

which will generate cross sections $\sigma_{R}^{\mathrm{SD}}(C)$ and $\sigma_{R}^{\mathrm{SD}}(N)$. Therefore, for a one-valence nucleon + core projectile, labeling the nucleon 1 ,

$$
S_{P}^{\mathrm{el}(2)}(b)=\left\langle\Phi_{0}^{(2)}\left|S_{C}^{\mathrm{SD}}\left(b_{C}\right) S_{N}^{\mathrm{SD}}\left(b_{1}\right)\right| \Phi_{0}^{(2)}\right\rangle,
$$

and, for a two-valence nucleon + core system,

$$
S_{P}^{\mathrm{el}(3)}(b)=\left\langle\Phi_{0}^{(3)}\left|S_{C}^{\mathrm{SD}}\left(b_{C}\right) S_{N}^{\mathrm{SD}}\left(b_{1}\right) S_{N}^{\mathrm{SD}}\left(b_{2}\right)\right| \Phi_{0}^{(3)}\right\rangle .
$$

This is the model used here. We note that the required physical inputs are theoretical few-body wave functions for the relative motions of the projectile constituents and the coretarget and valence nucleon-target elastic $S$ matrices. At the energies of the present analysis, several hundred $\mathrm{MeV} /$ nucleon, the latter are taken from the static density limit. These $S$ matrices must be consistent with experimental data for these independent binary systems at the same incident energy/nucleon. Such data currently comprise the reaction cross sections, but the $S$ matrices could be more stringently tested if elastic differential cross section angular distributions were also available. Our choice of $N N$ cross sections, $\vec{\sigma}_{N N}^{i T}$ ( $i=P, C, N)$, used in the static density calculations will be discussed later. In addition one often replaces $\vec{\sigma}_{N N}^{i T}$ by $\vec{\sigma}_{N N}^{i T}(1-i \alpha)$, with $\alpha$ the ratio of the real and imaginary parts of the forward scattering $N N$ amplitude, to account for the fact that the $N N$ amplitude is not entirely absorptive, even at high energy $[18,20]$. While the structure of the static density $S$ matrices are such that this modification has no effect on static density cross section calculations, the foldings present in Eqs. (12) and (13) mean that calculations based on the few-body description are sensitive, in principle, to such a modification. We comment upon this sensitivity in the results section.

The connection between calculated cross sections and projectile matter radii is transparent in the static density approximation, through the associated $\rho_{P}(r)$ entering Eq. (8). The connection in the few-body method is however less direct and the matter radii must be computed separately from the few-body wave function together with an assumed core nucleus matter distribution. The structure of the two- and three-body wave functions used in evaluating these expressions are outlined in the next section.

Because of the folding over the internal wave function of the projectile in Eqs. (12) and (13), the contributions of the valence and core particles to the calculated cross section are not readily decoupled. In the static density limit however, since the projectile density appears in an exponential function then, upon separating the core and valence particle contributions to the projectile density, i.e., $\rho_{P}(r)=\hat{\rho}_{C}(r)+\rho_{v}(r)$, one can decompose the integrand of the reaction cross section in Eq. (1) as [21]

$$
b\left[1-T_{P}^{\mathrm{SD}}(b)\right]=b\left[1-\hat{T}_{C}^{\mathrm{SD}}(b)\right]+b \hat{T}_{C}^{\mathrm{SD}}(b)\left[1-T_{v}^{\mathrm{SD}}(b)\right] .
$$

In this expression the transparency $\hat{T}_{C}^{\mathrm{SD}}$ due to $\hat{\rho}_{C}$, includes the effects of the convolution of the intrinsic core density $\rho_{C}$ with that for its center of mass motion in the projectile and is defined in the following section. $\hat{T}_{C}^{\mathrm{SD}}$ is not therefore the transparency of the free core-target system and will not generate the free core-target reaction cross section. The decomposition is nevertheless helpful in clarifying the importance of the valence nucleon contributions to the reaction cross section and we shall later compare the deviations of the 
projectile-target cross section integrands, calculated in the static density and few-body approaches, from this core contribution $b\left[1-\hat{T}_{C}^{\mathrm{SD}}\right]$ to elucidate the essential differences in the results of the two calculations.

\section{WAVE FUNCTION MODELS}

Here we discuss the general structure of the wave functions used. Calculations which use specific or published wave function models will refer clearly to the model in question in the later text.

\section{A. Three-body projectile systems}

We consider the two-neutron halo nuclei ${ }^{6} \mathrm{He},{ }^{11} \mathrm{Li}$, and ${ }^{14} \mathrm{Be}$ as three-body (core $+n+n$ ) systems, the main approximation being to neglect explicit consideration of the internal degrees of freedom of the 4-, 9-, and 12-nucleon cores. These are treated approximately in the model calculations through the use of phenomenological nucleon-core effective interactions. The total wave functions are the sum of the three Faddeev components, $\Phi_{0}^{(3)}=\Phi_{12}+\Phi_{C 1}+\Phi_{C 2}$, where each component is labeled by the interacting particle pair. Antisymmetrization implies that $\Phi_{C 2}$ and $\Phi_{C 1}$ are related by permutation of labels 1 and 2 . Following the notation of [22],

$\Phi_{0}^{(3)}=\Phi_{12}\left(\vec{r}_{12(C)}, \vec{r}_{12}\right)+\left(1+P_{12}\right) \Phi_{C 1}\left(\vec{r}_{C 1}, \vec{r}_{(C 1) 2}\right)$.

The total wave function $\Phi_{0}^{(3)}$ can be transformed into either set of coordinates, so that

$$
\Phi_{0}^{(3)}=\bar{\Phi}_{12}^{(3)}\left(\vec{r}_{12(C)}, \vec{r}_{12}\right)=\bar{\Phi}_{C 1}^{(3)}\left(\vec{r}_{C 1}, \vec{r}_{(C 1) 2}\right),
$$

where $\Phi_{0}^{(3)}$ and each $\bar{\Phi}^{(3)}$ has unit normalization.

For the purposes of the static density calculations, Eq. (8), and to compute the projectile rms radius, we require the projectile single-particle density corresponding to these Faddeev wave functions. This can be written

$$
\rho_{P}(r)=\hat{\rho}_{C}(r)+\rho_{v}(r),
$$

where $\hat{\rho}_{C}(r)$ and $\rho_{v}(r)$ are the contributions from the core and valence neutrons in the center of mass of the projectile. It follows that for a mass $A$ system the two-valence neutron density is

$$
\rho_{v}(\vec{r})=2\left(\frac{A}{A-1}\right)^{3} \int d \vec{r}_{C 1}\left|\bar{\Phi}_{C 1}^{(3)}\left(\vec{r}_{C 1}, \frac{A}{A-1} \vec{r}\right)\right|^{2},
$$

and, assuming a core internal density $\rho_{C}(r)$, then $\hat{\rho}_{C}(r)$ is obtained by folding with $\rho_{\text {c.m. }}(r)$, the distribution for the motion of the core's own center of mass, i.e.,

$$
\hat{\rho}_{C}(\vec{r})=\int d \vec{x} \rho_{C}(\vec{r}-\vec{x}) \rho_{\text {c.m. } .}(\vec{x}),
$$

where

$$
\rho_{\text {c.m. }}(\vec{r})=\left(\frac{A}{2}\right)^{3} \int d \vec{r}_{12}\left|\bar{\Phi}_{12}^{(3)}\left(\frac{A}{2} \vec{r}, \vec{r}_{12}\right)\right|^{2}
$$

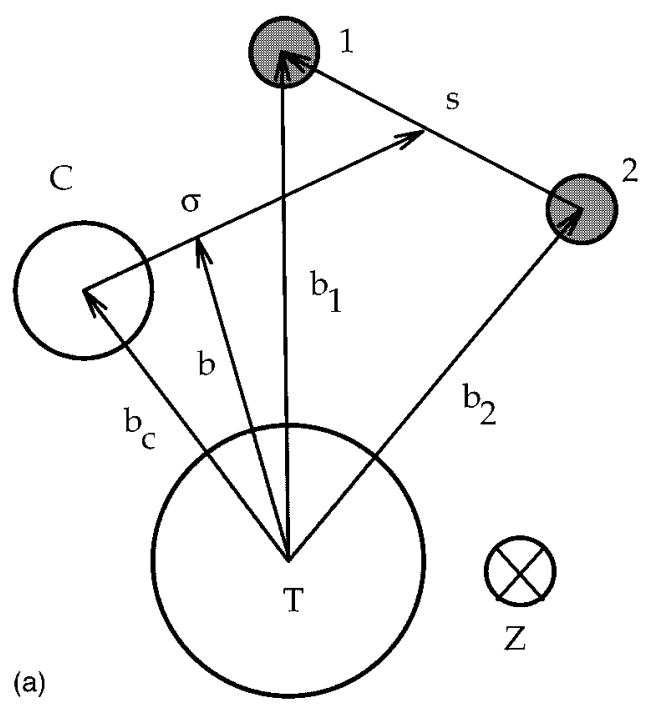

(a)

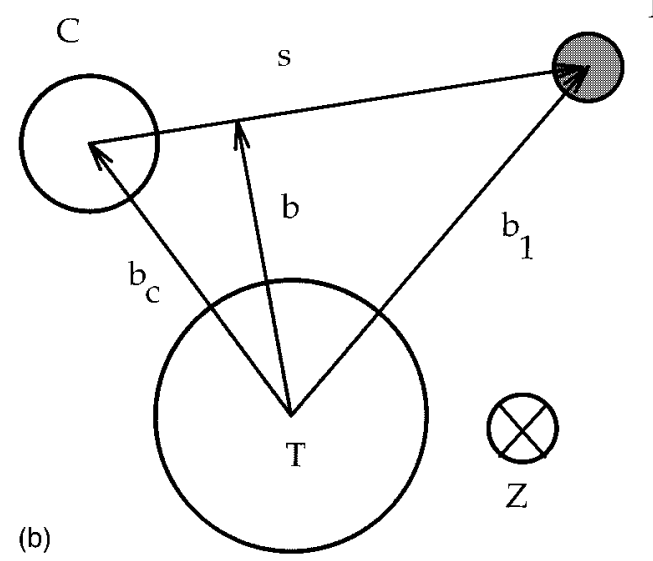

FIG. 3. Definition of position coordinates, in the plane perpendicular to the beam direction, in the case of (a) a three-body (twovalence nucleon + core) projectile and (b) a two-body (one-valence nucleon + core) projectile.

Where matter rms radii of three-body systems are quoted, they are calculated from the projectile ground state density Eq. (17) and thus assume a given core density distribution $\rho_{C}(r)$.

For the few-body $S$-matrix calculations from Eq. (13) on the other hand, we require $\left\langle\left|\bar{\Phi}_{12}^{(3)}\left(\vec{r}_{12(C)}, \vec{r}_{12}\right)\right|^{2}\right\rangle_{\text {spin }}$ where the bra-ket notation indicates that we sum only over the twoneutron spin variables. Details of the angular momentum structure of the three-body wave function and the probability density, and of its expression in terms of cylindrical polar coordinates to facilitate the integrations over impact parameters, can be found in Ref. [11]. Explicitly,

$$
S_{P}^{\mathrm{el}(3)}(b)=\int d^{2} \vec{\sigma} \int d^{2} \vec{s} \xi^{(3)}(\vec{\sigma}, \vec{s}) S_{C}^{\mathrm{SD}}\left(b_{C}\right) S_{N}^{\mathrm{SD}}\left(b_{1}\right) S_{N}^{\mathrm{SD}}\left(b_{2}\right),
$$

where $\vec{\sigma}$ and $\vec{s}$ are the components of the vectors $\vec{r}_{12(C)}$ and $\vec{r}_{12}$ in the plane normal to the incident beam direction, see Fig. 3(a), and the $z$-integrated probability density 


$$
\xi^{(3)}(\vec{\sigma}, \vec{s})=\int_{-\infty}^{\infty} d z_{12(C)} \int_{-\infty}^{\infty} d z_{12}\left\langle\left|\bar{\Phi}_{12}^{(3)}\left(\vec{r}_{12(C)}, \vec{r}_{12}\right)\right|^{2}\right\rangle_{\text {spin }}
$$

is written explicitly in Eqs. (28)-(31) of [11].

\section{B. Two-body projectile systems}

We also consider the one-neutron halo nucleus ${ }^{11} \mathrm{Be}$ $\left({ }^{10} \mathrm{Be}+n\right)$. Wave functions for this system are derived from two sources. In the first, simple $1 s_{1 / 2}{ }^{10} \mathrm{Be}+n$ cluster model wave functions of the ${ }^{11} \mathrm{Be}$ ground state are constructed as bound states in Woods-Saxon potential wells of differing geometries, the potential strength being adjusted to reproduce the empirical ${ }^{10} \mathrm{Be}+n$ separation energy. Such a model is flexible and simple and allows one to generate ${ }^{11} \mathrm{Be}$ structures with a range of matter rms radii. Our second source of two-body wave functions is from more microscopic coupled channels bound state calculations which include core excitation degrees of freedom [23]. These calculations predict

${ }^{11} \mathrm{Be}$ level spectra and spectroscopic amplitudes in addition to the ground state wave functions and also make specific predictions for the rms matter radius of the ground state.

Using wave functions from either source, for the static density calculations, Eq. (8), we need to evaluate the projectile density. As with the three-body systems, the neutroncore relative motion wave functions are used to construct the valence particle density component, and to fold the free core density $\rho_{C}(r)$. Writing $\rho_{P}(r)=\hat{\rho}_{C}(r)+\rho_{1}(r)$ then $\hat{\rho}_{C}(r)$ is given by Eq. (19) but where now

$$
\begin{gathered}
\rho_{1}(\vec{r})=\left[\frac{A}{A-1}\right]^{3}\left|\Phi_{0}^{(2)}\left(\frac{A}{A-1} \vec{r}\right)\right|^{2}, \\
\rho_{\text {c.m. }}(\vec{r})=A^{3}\left|\Phi_{0}^{(2)}(A \vec{r})\right|^{2} .
\end{gathered}
$$

For the few-body $S$-matrix calculations, Eq. (12), we require $\left\langle\left|\Phi_{0}^{(2)}\left(\vec{r}_{1 C}\right)\right|^{2}\right\rangle_{\text {spin }}$ where, in common with the notation for the three-body projectiles, we have summed over the nucleon and core spin variables. Now

$$
S_{P}^{\mathrm{el}(2)}(b)=\int d^{2} \vec{s} \xi^{(2)}(\vec{s}) S_{C}^{\mathrm{SD}}\left(b_{C}\right) S_{N}^{\mathrm{SD}}\left(b_{1}\right),
$$

where $\vec{s}$ is the component of $\vec{r}_{1 C}$ in the plane normal to the incident beam, Fig. 3(b), and

$$
\xi^{(2)}(\vec{s})=\int_{-\infty}^{\infty} d z_{1 C}\left\langle\left|\Phi_{0}^{(2)}\left(\vec{r}_{1 C}\right)\right|^{2}\right\rangle_{\text {spin }} .
$$

\section{CALCULATIONS}

We consider the calculated cross sections and deduced rms matter radii for the one-neutron halo nucleus ${ }^{11} \mathrm{Be}$ and the two-neutron halo nuclei ${ }^{6} \mathrm{He},{ }^{11} \mathrm{Li}$, and ${ }^{14} \mathrm{Be}$. A consideration of ${ }^{8} \mathrm{~B}$, a candidate for a one-proton halo structure, can be found in [4]. In common with previous analyses of the experimental data we calculate reaction cross sections and make comparison with the experimental interaction cross sections. This procedure has been shown to be accurate for halo nuclei [15].
TABLE I. Core and halo nucleus matter rms radii deduced from the static density (SD) and few-body (FB) models, respectively. The references are to the experimental interaction cross section data, all at $800 \mathrm{MeV} /$ nucleon.

\begin{tabular}{lcccc}
\hline \hline Nucleus & rms radius $(\mathrm{fm})$ & $\sigma_{I}(\mathrm{expt})(\mathrm{mb})$ & Ref. & Method \\
\hline${ }^{4} \mathrm{He}$ & $1.58 \pm 0.04$ & $503 \pm 5$ & {$[2]$} & $\mathrm{SD}$ \\
${ }^{6} \mathrm{He}$ & $2.71 \pm 0.04$ & $722 \pm 5$ & {$[2]$} & FB (free $\left.{ }^{4} \mathrm{He}\right)$ \\
& $2.69 \pm 0.04$ & & & FB (scaled $\left.{ }^{4} \mathrm{He}\right)$ \\
${ }^{7} \mathrm{Be}$ & $2.31 \pm 0.05$ & $738 \pm 9$ & {$[1]$} & $\mathrm{SD}$ (from [4]) \\
${ }^{8} \mathrm{~B}$ & $2.50 \pm 0.04$ & $798 \pm 6$ & {$[35]$} & FB (from [4]) \\
${ }^{9} \mathrm{Li}$ & $2.30 \pm 0.02$ & $796 \pm 6$ & {$[1]$} & $\mathrm{SD}$ \\
${ }^{11} \mathrm{Li}$ & $3.53 \pm 0.10$ & $1060 \pm 10$ & {$[16]$} & $\mathrm{FB}$ \\
${ }^{10} \mathrm{Be}$ & $2.28 \pm 0.02$ & $813 \pm 10$ & {$[1]$} & $\mathrm{SD}$ \\
${ }^{11} \mathrm{Be}$ & $2.90 \pm 0.05$ & $942 \pm 8$ & {$[3]$} & $\mathrm{FB}$ \\
${ }^{12} \mathrm{Be}$ & $2.54 \pm 0.05$ & $927 \pm 18$ & {$[3]$} & $\mathrm{SD}$ \\
${ }^{14} \mathrm{Be}$ & $3.20 \pm 0.30$ & $1109 \pm 69$ & {$[29]$} & FB \\
\hline \hline
\end{tabular}

\section{A. Static density calculations}

We first apply the formalism developed above to calculate, in static density approximation, the elastic $S$ matrices, $S_{C}^{\mathrm{SD}}$ and $S_{N}^{\mathrm{SD}}$ and the reaction cross sections, $\sigma_{R}^{\mathrm{SD}}(C)$ and $\sigma_{R}^{\mathrm{SD}}(N)$, for all the required core- and nucleon-target subsystems. The $S$ matrices are then input to the few-body calculations for the one- and two-neutron halo nuclei. The core nucleus cross sections $\sigma_{R}^{\mathrm{SD}}(C)$ are required to determine the core matter rms radii consistently. For all projectile nuclei we initially consider reactions on a ${ }^{12} \mathrm{C}$ target at $800 \mathrm{MeV} /$ nucleon. The choice of energy and target is dictated by our wish to validate the theoretical description of each binary subsystem by comparison with experiment. For comparison later with the few-body calculations, and for comparison with earlier work, we also calculate the composite (halo) nucleus cross sections $\sigma_{R}^{\mathrm{SD}}(P)$ within the static density approximation.

For all these static density calculations we use the parametrization of the free $N N$ cross section, $\vec{\sigma}_{N N}^{i T}(i=P, C, N)$, of Charagi and Gupta [24]. A Gaussian matter distribution is assumed for ${ }^{12} \mathrm{C}$ in all cases with rms matter radius $\left\langle r^{2}\right\rangle_{T}^{1 / 2}=2.32 \mathrm{fm}[3]$.

With these inputs, and assuming Gaussian matter distributions for the core nuclei, we find the core radii $\left\langle r^{2}\right\rangle_{4}^{1 / 2}=1.58 \mathrm{fm},\left\langle r^{2}\right\rangle_{9}^{1 / 2}=2.30 \mathrm{fm},\left\langle r^{2}\right\rangle_{10}^{1 / 2}=2.28 \mathrm{fm}$, and $\left\langle r^{2}\right\rangle_{12}^{1 / 2}=2.54 \mathrm{fm}$, generate reaction cross sections for the core-target subsystems [7] of $\sigma_{R}^{\mathrm{SD}}\left({ }^{4} \mathrm{He}\right)=503(503 \pm 5) \mathrm{mb}$, $\sigma_{R}^{\mathrm{SD}}\left({ }^{9} \mathrm{Li}\right)=796(796 \pm 6) \mathrm{mb}, \sigma_{R}^{\mathrm{SD}}\left({ }^{10} \mathrm{Be}\right)=813 \quad(813 \pm 10)$ $\mathrm{mb}$, and $\sigma_{R}^{\mathrm{SD}}\left({ }^{12} \mathrm{Be}\right)=927(927 \pm 18) \mathrm{mb}$. The empirical values, in parentheses, are taken from a number of references. These values and references are collected in Table I. These deduced core radii agree with those of Tanihata et al. [3] within error bars. The calculated nucleon- ${ }^{12} \mathrm{C}$ cross section at $800 \mathrm{MeV}, \sigma_{R}^{\mathrm{SD}}(N)=231 \mathrm{mb}$, also agrees with experiment [17] within quoted errors. Thus the calculated $S$ matrices for each projectile constituent-target system, and the core nucleus sizes input to the few-body calculations, are each consistent with available empirical data for that binary system. 


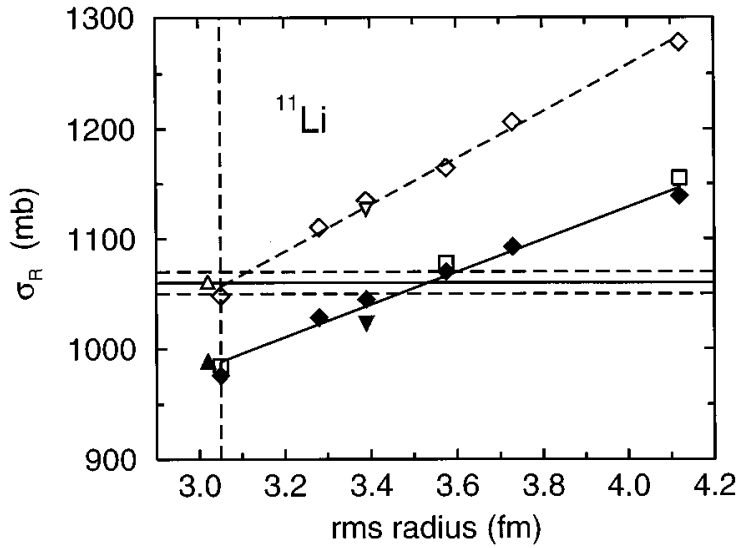

FIG. 4. Calculated static density and few-body reaction cross sections at $800 \mathrm{MeV} /$ nucleon incident energy for ${ }^{11} \mathrm{Li}$ projectiles as a function of projectile rms matter radius, for a ${ }^{12} \mathrm{C}$ target. The lines and symbols are described in the text.

\section{B. Few-body calculations for two-neutron halo nuclei}

\section{Calculations for ${ }^{11} \mathrm{Li}$}

Figure 4 shows the results of static density and few-body calculations for ${ }^{11} \mathrm{Li}+{ }^{12} \mathrm{C}$ using a number of ${ }^{11} \mathrm{Li}$ wave functions. We show the calculated cross sections versus the matter rms radii calculated from the wave functions. The horizontal band shows the experimental interaction cross section datum $\sigma\left({ }^{11} \mathrm{Li}\right)=1060 \pm 10 \mathrm{mb}[16]$ and the vertical dashed line the matter radius $\left\langle r^{2}\right\rangle_{11}^{1 / 2}=3.05 \mathrm{fm}$ obtained if we adopt the static density approximation and a Gaussian density for the projectile; in the spirit of $[3,7]$. The value is within error bars of the previously quoted value $\left\langle r^{2}\right\rangle_{11}^{1 / 2}=3.10 \pm 0.17 \mathrm{fm}[3]$.

The (lower) full symbols and open squares are the results of the few-body calculations. The (upper) open symbols are the results of the static density calculations using the ground state density calculated from the same wave function models. The rms radii are computed assuming the core matter densities deduced above. The reduction in the calculated cross sections, or increased transparency of the projectile in the former case, is immediately evident. From left to right the diamond symbols correspond to the P0 through P4 intruder $s$-wave (Faddeev) model wave functions of Thompson and Zhukov [25], with increasing rms radius. The extreme righthand point is a continuation of these model wave functions (P5) with a $1 s$-state scattering length of $-44 \mathrm{fm}$ and an $80 \%$ $\left(1 s_{1 / 2}\right)^{2}$ probability. The upright triangles denote calculations using the L6A pairing model wave function [26] (with rms radius $3.02 \mathrm{fm}$ ) which, in the static density picture, reproduces the experimental cross section. The inverted triangles are the predictions of the $0 s$-wave intruder wave function (denoted $G 1$ in [25]), generated in a very shallow binding potential, from the early work of Johannsen, Jensen, and Hansen [27]. The straight lines through these different wave function models are linear least squares fits to the theoretical calculations.

The difference between the two approaches is large. The static density calculations, even using Faddeev wave functions, suggest a matter rms radius of order $3.1 \mathrm{fm}$, as reported previously. A correct treatment of the ${ }^{11} \mathrm{Li}$ three-body char-

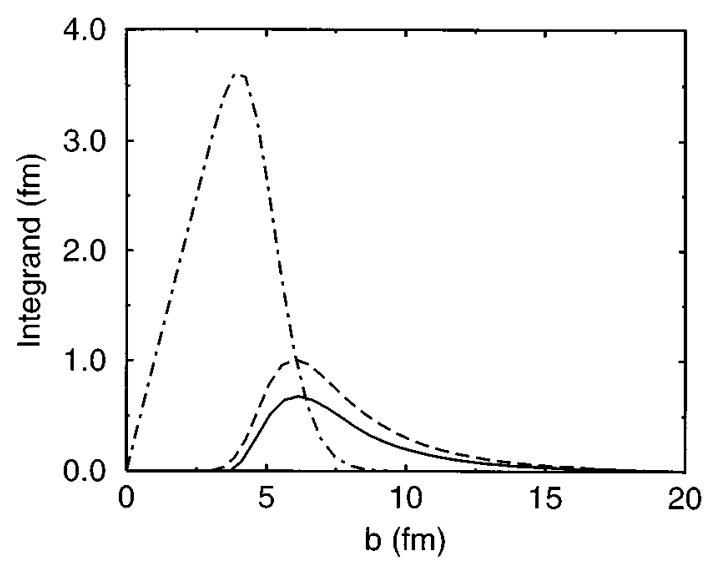

FIG. 5. Calculated integrands of the reaction cross section impact parameter integral from the static density and few-body analyses, using the $\mathrm{P} 3{ }^{11} \mathrm{Li}$ wave function, for the reaction on a ${ }^{12} \mathrm{C}$ target at $800 \mathrm{MeV} /$ nucleon. The dot-dashed curve shows the core contribution and the solid and dashed curves the valence contributions in the few-body and static density calculations, respectively.

acter, however, now suggests a more extended halo $\left\langle r^{2}\right\rangle_{11}^{1 / 2} \approx 3.53 \pm 0.10 \mathrm{fm}$, in the middle of the band of values generated by intruder state wave function models. Such wave functions, with significant $s$-wave admixtures in their ground state, lead naturally to a more extended matter distribution.

As was discussed in Sec. II, a consideration of the fact that the $N N$ amplitude is not entirely imaginary at high energy has no implications for the static density cross section calculations. Replacing $\bar{\sigma}_{N N}^{i T}$ by $\bar{\sigma}_{N N}^{i T}(1-i \alpha)$ will however affect the cross sections calculated in the few-body description where the binary channel $S$ matrices are multiplied and averaged over the projectile wave function before the square modulus is taken. We have therefore repeated the few-body calculations for the P0, P3, and P5 wave functions using the prescription for the $N N$ amplitude of Ray (Table I of [20]) with a nonvanishing, energy dependent, $\alpha$. The isospin average of the tabulated $\alpha \vec{\sigma}_{N N}^{i T}$ appropriate for the ${ }^{9} \mathrm{Li}$ and neutron-target systems is taken. We obtain results for these wave functions shown by the open square symbols in Fig. 4, with changes in the calculated cross sections of less than 8 $\mathrm{mb}$, and only very minor effects for deduced radii. We return to this effect in considering the energy dependence of the ${ }^{11} \mathrm{Li}$ cross section.

To clarify the origin of the increased transparency of the collision process, we show in Fig. 5 the integrands of the cross section expression, Eq. (1), from the static density and few-body calculations. The P3 wave function of [25] is used in these calculations. As discussed earlier, there is no way to extract precisely the core and valence particle contributions to the cross section in the few-body model. To provide insight into these roles however we plot the quantities $I_{C}=b\left[1-\hat{T}_{C}^{\mathrm{SD}}\right]$ (dot-dashed curve), the static density "core", component discussed in Sec. II, and the quantities $b\left[1-\left|S_{P}^{\mathrm{SD}}\right|^{2}\right]-I_{C}$ (dashed curve) and $b\left[1-\left|S_{P}^{\mathrm{el}(3)}\right|^{2}\right]-I_{C}$ (solid curve), being the remainder of the integrand in the static density and the few-body approaches, respectively. These remainders will be identified with the valence particle contributions. Their localization to large impact parameters suggests they do indeed represent dominantly valence par- 


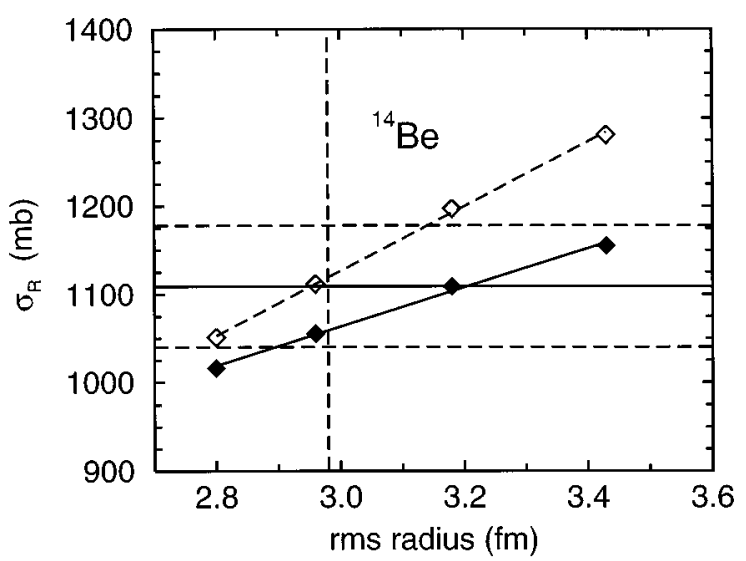

FIG. 6. As for Fig. 4 but for ${ }^{14} \mathrm{Be}$ projectiles.

ticle effects. Figure 5 shows clearly the increased transparency of the halo resulting from the more accurate treatment of the correlated three-body structure of ${ }^{11} \mathrm{Li}$. The contribution to the cross section from $I_{C}$ is $866.4 \mathrm{mb}$, to be compared with the free ${ }^{9} \mathrm{Li}$ value of $796 \mathrm{mb}$. The valence contribution is $305 \mathrm{mb}$ in the static density approach. In the few-body calculation the valence contribution is reduced by $35 \%$ with respect to this static density value, generating only $200 \mathrm{mb}$.

\section{Calculations for ${ }^{14} \mathrm{Be}$}

Figure 6 shows the results of static density and few-body calculations for the ${ }^{14} \mathrm{Be}+{ }^{12} \mathrm{C}$ system at $800 \mathrm{MeV} /$ nucleon. As in Fig. 4, we show the calculated cross sections versus the matter rms radii of a series of theoretical wave function models [28]. The horizontal band shows the experimental interaction cross section datum $\sigma\left({ }^{14} \mathrm{Be}\right)=1109 \pm 69 \mathrm{mb}$ [29] which currently has large experimental uncertainty. The vertical dashed line shows the rms matter radius we obtain, $\left\langle r^{2}\right\rangle_{14}^{1 / 2}=2.98 \mathrm{fm}$, if we adopt the static density approximation and assume a Gaussian density distribution for the projectile; in the spirit of $[3,7]$.

The (lower) full symbols are the results of the few-body calculations for each wave function model. The (upper) open symbols are the results of the static density calculations using the projectile ground state density calculated from the same wave function models. The results again show the expected reduction in the calculated cross sections, or increased transparency of the projectile, in the latter case. From left to right the diamond symbols correspond to the $\mathrm{C} 4, \mathrm{C} 7, \mathrm{D} 4$, and C9 Faddeev wave functions of Thompson and Zhukov [28]. The results are very similar, qualitatively, to the ${ }^{11} \mathrm{Li}$ case but somewhat smaller in magnitude due to the increased empirical (1.31 MeV [30]) and theoretical two-neutron separation energy for ${ }^{14} \mathrm{Be}$ in the wave functions of [28]. It is clear that the experimental datum does not currently permit an accurate determination of the projectile matter radius. We estimate $\left\langle r^{2}\right\rangle_{14}^{1 / 2}=3.20 \pm 0.30 \mathrm{fm}$. We note however that, for cross sections in the range of the current experimental error bars, the few-body calculations result in a deduced rms matter radius greater than that of the corresponding static density value by up to $0.35 \mathrm{fm}$.

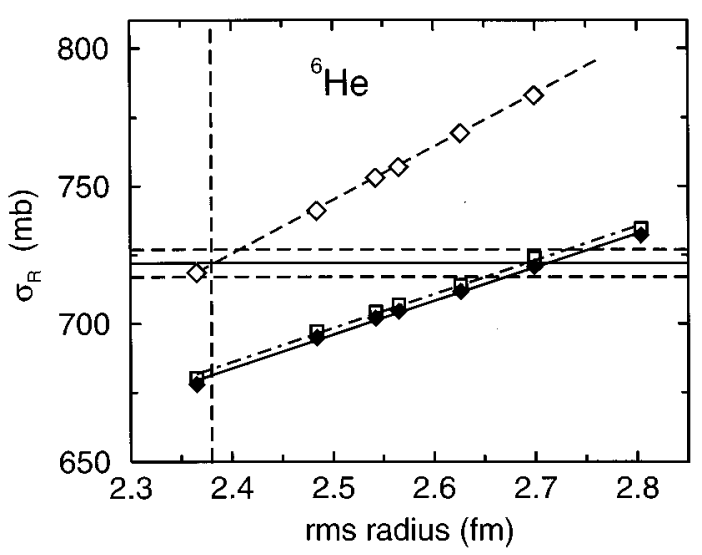

FIG. 7. As for Fig. 4 but for ${ }^{6} \mathrm{He}$ projectiles. The open square points and dot-dashed line are the few-body calculations when the core size is scaled by $1.5 \%$ in the calculation of the core-target static density $S$ matrix.

\section{Calculations for ${ }^{6} \mathrm{He}$}

Figure 7 shows the static density and few-body calculations for the ${ }^{6} \mathrm{He}+{ }^{12} \mathrm{C}$ system at $800 \mathrm{MeV} /$ nucleon, presented as in Figs. 4 and 6 . The horizontal band shows the reported cross section datum $\sigma\left({ }^{6} \mathrm{He}\right)=722 \pm 5 \mathrm{mb}$ [31]. The vertical dashed line shows the rms matter radius we obtain, $\left\langle r^{2}\right\rangle_{6}^{1 / 2}=2.38 \mathrm{fm}$, if we adopt the static density approximation and assume a single Gaussian density distribution for the three-body projectile.

The (lower) full diamond and open square symbols show the few-body calculations and the (upper) open diamond symbols the static density calculations using the projectile ground state density calculated from the same wave function. The few-body results are again reduced relative to the static density model. From left to right the diamond symbols correspond to a representative selection of the Faddeev wave function models of Table II, models P1, GB3, FC, FC6, FB, $\mathrm{K}$, and $\mathrm{C}$, in order of increasing rms radius, calculated using the methods described in [14]. As for all systems considered, we compute these ${ }^{6} \mathrm{He}$ matter radii assuming a core matter radius of $\left\langle r^{2}\right\rangle_{4}^{1 / 2}=1.58 \mathrm{fm}$, consistent with the calculated $\sigma_{R}^{\mathrm{SD}}\left({ }^{4} \mathrm{He}\right)$ and the data.

The wave functions shown span the full range of rms radii. Those not plotted lie on the same straight lines. The wave functions are all orthogonal to an occupied $0 s$ eigenstate assumed to be at $-20 \mathrm{MeV}$ in the $n-{ }^{4} \mathrm{He}$ interaction. We include now the results of using parity-dependent $n-{ }^{4} \mathrm{He}$ interactions derived [32] from inverse scattering methods to $n-{ }^{4} \mathrm{He}$ elastic scattering data at around $1.2 \mathrm{MeV}$ (P1 and P3) and over the range $0-20 \mathrm{MeV}$ (Q1 and Q3). A common feature of all the remaining three-body calculations is that using interactions consistent with elastic scattering leads to larger and slightly too weakly bound structures. The $\mathrm{C}$ model uses the Woods-Saxon potential of [33], and the $\mathrm{K}$ model the Gaussian potential of [34].

To approach the empirical three-body binding of 0.97 $\mathrm{MeV}$, these models all require adjustment of the $n-{ }^{4} \mathrm{He}$ interaction from that which reproduces free $n-{ }^{4} \mathrm{He}$ elastic scattering. The way chosen to do this was to increase the range of the $n-\alpha$ core interaction (e.g., by $2 \%$ and $2.5 \%$ for GB3 and $\mathrm{FC}$, respectively) to account for possible core polariza- 
TABLE II. Different ${ }^{6} \mathrm{He}$ ground state wave function models, rms radii, and two-neutron separation energies $E(2 n)$. The $n-n$ potentials used are the super soft core $[\mathrm{SSC}(\mathrm{C})]$ [36], Reid soft core (RSC) [37], and $s$-wave Gaussian (G) [38] interactions. The $n-\alpha$ potentials used are two-parity $(\pi)$-dependent inverse-scattering potentials [32] obtained from two energy ranges $(P: 1.2 \mathrm{MeV}$ and $Q: 0-20 \mathrm{MeV}$ ), the Woods-Saxon (WS) potential of [33], and the $\ell$-dependent Gaussian (SBB) potential of [34]. rms radii are computed assuming a ${ }^{4} \mathrm{He}$ core radius of $1.58 \mathrm{fm}$ of Table I.

\begin{tabular}{lcccccc}
\hline \hline Model & $V_{n n}$ & $\mathrm{~V}_{n \alpha}$ & $\begin{array}{c}r_{n \alpha} \text { incr. } \\
(\%)\end{array}$ & $\ell_{n \alpha}$ & $\begin{array}{c}E(2 n) \\
(\mathrm{MeV})\end{array}$ & $\begin{array}{c}\text { rms radius } \\
(\mathrm{fm})\end{array}$ \\
\hline P3 & $\mathrm{SSC}(\mathrm{C})$ & $\pi$ dep. $(P)$ & 0 & $s, p, d$ & -1.21 & 2.34 \\
P1 & $\mathrm{SSC}(\mathrm{C})$ & $\pi$ dep. $(P)$ & 0 & $s, p$ & -1.15 & 2.36 \\
GB3 & $\mathrm{G}$ & WS & 2 & $s, p$ & -1.00 & 2.49 \\
FC & $\mathrm{SSC}(\mathrm{C})$ & WS & 2.5 & $s, p$ & -0.93 & 2.53 \\
FC6 & $\mathrm{SSC}(\mathrm{C})$ & WS & 2.5 & $s, p, d$ & -0.93 & 2.56 \\
Q3 & $\mathrm{SSC}(\mathrm{C})$ & $\pi$ dep. $(Q)$ & 0 & $s, p, d-0.70$ & 2.58 \\
Q1 & $\mathrm{SSC}(\mathrm{C})$ & $\pi$ dep. $(Q)$ & 0 & $s, p$ & -0.65 & 2.60 \\
FB & $\mathrm{SSC}(\mathrm{C})$ & WS & 1.5 & $s, p$ & -0.63 & 2.62 \\
FA & $\mathrm{SSC}(\mathrm{C})$ & WS & 1.0 & $s, p$ & -0.49 & 2.67 \\
K & $\mathrm{RSC}$ & $\mathrm{SBB}$ & 0.0 & $s, p, d-0.42$ & 2.69 \\
C & $\mathrm{SSC}(\mathrm{C})$ & WS & 0.0 & $s, p$ & -0.21 & 2.80 \\
\hline \hline
\end{tabular}

tion effects. The values are shown in Table II. After such $\alpha$ scaling, all calculations with realistic potentials (with ${ }^{3} P_{1}$ repulsion) give matter radii around $2.55 \mathrm{fm}$. We calculate the reaction cross sections from all these models in order to have range of ${ }^{6} \mathrm{He}$ model wave functions with different matter radii, despite the variation of binding energies as shown in Table II.

In common with our earlier results, the static density calculations suggest a ${ }^{6} \mathrm{He}$ matter rms radius in agreement with that obtained using a Gaussian density distribution, that is of order $2.38 \mathrm{fm}$. Within the few-body framework, however, the Faddeev wave functions discussed above now suggest a ${ }^{6} \mathrm{He}$ matter radius of $2.71 \pm 0.04 \mathrm{fm}$. As for ${ }^{11} \mathrm{Li}$, this is larger than previous estimates, such as the value $2.57 \mathrm{fm}$ [8] deduced on the basis of a comparison of the ${ }^{6} \mathrm{He}$ and ${ }^{6} \mathrm{Li}$ systems. For ${ }^{11} \mathrm{Li}, s$-wave intruder models generate larger radii in a natural way. The configuration of the two neutrons in ${ }^{6} \mathrm{He}$, however, is thought to be essentially pure $\left(p_{3 / 2}\right)^{2}$, and the more extended wave functions in Table II are obtained only at the expense of underbinding of the three-body system.

The importance of the modification required to the $n-{ }^{4} \mathrm{He}$ interaction in the bound state, to generate sufficient binding in some cases, suggests that for ${ }^{6} \mathrm{He}$ there is some evidence of an associated core polarization effect. This being so, the core-target interaction entering the few-body description used here should also, in principle, be modified from that for free ${ }^{4} \mathrm{He}$-target scattering. To investigate this effect, of a modified core-target $S$ matrix, we have repeated calculations using the few-body model increasing the rms radius of the core density entering the static density calculation of $S_{C}^{\mathrm{SD}}$ Eq. (10), by $1.5 \%$. The resulting cross sections are shown by the open square symbols and the dot-dashed angled line in Fig. 7. The result is to increase the cross section by of order $3-5 \mathrm{mb}$, comparable to the error on the cross section datum, suggesting a slightly reduced matter radius of

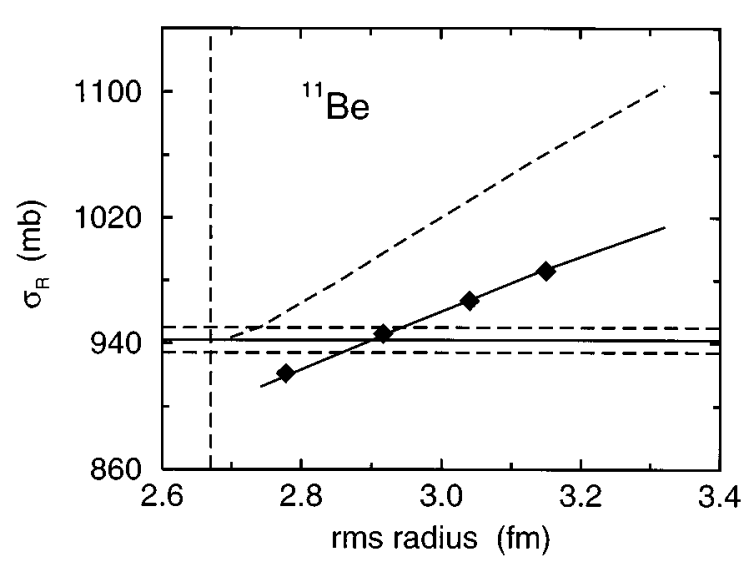

FIG. 8. As for Fig. 4 but for ${ }^{11}$ Be projectiles.

$2.69 \pm 0.04 \mathrm{fm}$. This value remains at the upper limit of values from the three-body models. It is clear however that the magnitude of the few-body corrections are significantly greater than effects arising from detailed consideration of the core size. The results are collected in Table I.

\section{Few-body calculations for one-neutron halo nuclei: ${ }^{11} \mathrm{Be}$}

Figure 8 shows the results of few-body and static density calculations for the one-neutron halo system ${ }^{11} \mathrm{Be}$. As previously, the horizontal band shows the experimental cross section datum $\sigma\left({ }^{11} \mathrm{Be}\right)=942 \pm 8 \mathrm{mb}[3]$ and the vertical dashed line the rms matter radius we obtain, $\left\langle r^{2}\right\rangle_{11}^{1 / 2}=2.67 \mathrm{fm}$, if we adopt the static density approximation and assume a Gaussian density for the projectile. This value is consistent with that of Ref. [3], i.e., $\left\langle r^{2}\right\rangle_{11}^{1 / 2}=2.71 \pm 0.05 \mathrm{fm}$. The results are qualitatively similar to those of the three-body cases. The angled dashed line shows the static density calculations and the angled solid line the few-body model results. Here, both of the lines connect calculations using simple two-body $\left(1 s_{1 / 2}\right)$ cluster wave functions for ${ }^{11} \mathrm{Be}$ using potentials with a range of geometries and depths adjusted to the observed neutron separation energy $0.503 \mathrm{MeV}$.

The solid symbols are the few-body calculations using ${ }^{11} \mathrm{Be}$ ground state wave functions [23] which include the effects of core $\left({ }^{10} \mathrm{Be}\right)$ deformation and excitation. These have been calculated using different underlying diagonal interactions for the neutron- ${ }^{10} \mathrm{Be}$ system so as to generate a family of structures with different radii for the purpose of the present analysis. We should point out however that the wave function model with rms radius of $2.92 \mathrm{fm}$, whose calculated cross section lies within experimental error bars, is that which best describes the excited state spectrum of ${ }^{11} \mathrm{Be}$.

These more microscopic wave functions generate cross sections which appear to follow precisely the trends of the inert core calculations. However, this is a little too simplistic and hides some important structure implications. In particular, the ground state wave functions of the coupled channels bound state models contain significant components in which the core is excited and the nucleon is in a $0 d_{5 / 2}$ orbital. Both of these effects reduce the contribution to the rms radius from this component of the wave function and hence the wave functions represented by the solid points are somewhat smaller than would be obtained using uncoupled calculations 


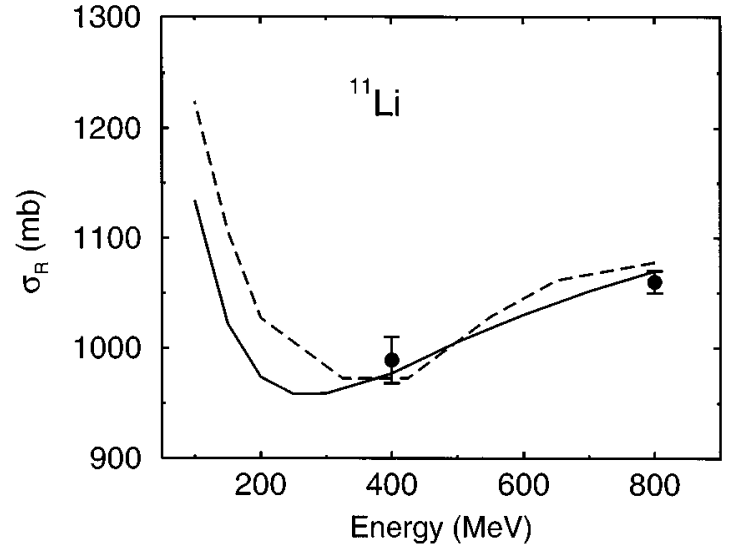

FIG. 9. Experimental and calculated reaction cross sections for ${ }^{11} \mathrm{Li}+{ }^{12} \mathrm{C}$ as a function of the incident energy/nucleon for the P3 wave function. The solid and dashed lines are the few-body calculations using the $N N$ cross section prescriptions of Charagi and Gupta [24] and Ray [20], respectively.

and the same underlying two-body interactions. Our calculations suggest a revised matter rms radius of $\left\langle r^{2}\right\rangle_{11}^{1 / 2}=2.90 \pm 0.05 \mathrm{fm}$. The radii obtained and used for all core and composite nuclei are collected in Table I.

\section{Energy dependence of the ${ }^{11} \mathrm{Li}$ cross section}

Reaction cross section data are available for the projectiles ${ }^{11} \mathrm{Li}$ and ${ }^{14} \mathrm{Be}$ at $400 \mathrm{MeV} /$ nucleon [29], which is also within the energy range in which our model is applicable. However, only the ${ }^{11} \mathrm{Li}$ data are of sufficient accuracy for a quantitative discussion. Within the few-body model, the only explicit energy dependence is that of the free $N N$ cross section which enters the calculation of all static density elastic $S$ matrices. In Fig. 9 we present the energy dependence of the ${ }^{11} \mathrm{Li}$ cross section calculated using the few-body model. The calculations use the P3 model wave function [25]. The solid curve shows the calculations using the $N N$ cross section parametrization of Charagi and Gupta [24]. The calculations show the energy dependence of the cross section in this energy regime to be naturally reproduced by the fewbody calculations.

When using the prescription for the $N N$ amplitude of Ray (Table I of [20]) we obtain the dashed curve. The agreement between the few-body calculations for the two $N N$ descriptions is good for energies in excess of $300 \mathrm{MeV} /$ nucleon. However the calculations begin to diverge at lower energies, showing sensitivity to the details of the description of the $N N$ amplitude. For this reason we do not extend the present analysis to lower energy, where additional data exist only below $100 \mathrm{MeV} /$ nucleon. While the adiabatic approximation is expected to be valid to energies of perhaps as low as 30 $\mathrm{MeV} /$ nucleon, the use of the static density approximation for the elastic $S$ matrices will certainly not be appropriate.

These calculations at high energy thus cannot yet be connected with calculations at lower energies where noneikonal corrections and account of the Coulomb interaction are essential, and where the required constituent-target elastic $S$ matrices might be better calculated from empirical optical potentials, fitted to elastic scattering angular distributions.
Additional experimental data in the energy region between 100 and $400 \mathrm{MeV} /$ nucleon will certainly be needed to clarify this connection.

\section{SUMMARY AND CONCLUSIONS}

To date, matter radii of light exotic nuclei have been deduced using approximations to Glauber theory which neglect particle correlations within the projectile and target. We have presented an alternative quantitative procedure for extracting such radii from cross section measurements at high energies. We have stressed the need for a description of the reaction which includes a proper consideration of the strong spatial correlations in halo nuclei implied by their effective fewbody character. Use of the adiabatic nature of the collision at high energy while retaining the static density approximation for the description of the interaction of the valence and core components of the projectile with the target leads to a practical alternative calculation of the composite projectile cross section.

A particularly attractive feature of this approach is that it makes clear the connection between the calculated cross section of the composite and (a) its theoretical few and/or manybody wave function (and not its single-particle density), and (b) empirical data for the scattering from the target of its few constituent bodies; through their associated elastic $S$ matrices. One can thus verify that each of these independent binary channel inputs to the composite projectile reaction mechanism are consistent with whatever experimental data are available for these systems.

We have reanalyzed experimental data of reaction cross sections for the two-neutron halo nuclei ${ }^{6} \mathrm{He},{ }^{11} \mathrm{Li}$, and ${ }^{14} \mathrm{Be}$ and the representative one-neutron halo system ${ }^{11} \mathrm{Be}$, all on a ${ }^{12} \mathrm{C}$ target at $800 \mathrm{MeV} /$ nucleon. We have also presented calculations at $400 \mathrm{MeV} /$ nucleon for the two-neutron halo projectile ${ }^{11} \mathrm{Li}$. By use of the adiabatic treatment of the internal coordinates, we have incorporated realistic two- and three-body wave functions for these projectiles. We have compared the few-body and static density calculations of reaction cross sections for these systems. We have shown that the granular structure of the projectiles implied by realistic few-body wave functions reduces considerably the calculated reaction cross sections and thus increases significantly the values of matter rms radii deduced from experimental data, compared to those from static density estimates. By reference to the integrands of the cross sections, we have shown this to result from a very significant increase, $35 \%$ in the case of ${ }^{11} \mathrm{Li}$, in the transparency of the valence nucleon component of the cross section as a result of few-body correlations.

We deduce matter rms radii for ${ }^{11} \mathrm{Li}$ and ${ }^{11} \mathrm{Be}$ of $3.53 \pm 0.10 \mathrm{fm}$ and $2.90 \pm 0.05 \mathrm{fm}$, respectively, increases of $14.0 \%$ and $7 \%$ over previously tabulated values. Our radius for ${ }^{11} \mathrm{Li}$ is now consistent with models with a significant $1 s$-wave intruder state component. Our radius for ${ }^{11} \mathrm{Be}$ is consistent with models which include core excitation and reorientation. We present cross sections for a range of theoretical models for the ${ }^{14} \mathrm{Be}$ structure. The error on the available cross section measurement for this system takes in all these models but we estimate $\left\langle r^{2}\right\rangle_{14}^{1 / 2}=3.20 \pm 0.30 \mathrm{fm}$. Calculations show that the few-body description generates a matter radius greater than static density estimates by up to $0.35 \mathrm{fm}$. 
For ${ }^{6} \mathrm{He}$, using the static density $S$ matrix for free ${ }^{4}$ He-target scattering, few-body calculations suggest an rms radius of $2.71 \pm 0.04 \mathrm{fm}$ from the reported cross section measurement. This rms radius can be obtained from current theoretical models only at the expense of underbinding the threebody system. Taking account of a possible scaling of the core size in this system, by of order $1.5 \%$, required in some structure calculations to bind ${ }^{6} \mathrm{He}$ appropriately, does not significantly reduce this deduced value. For ${ }^{6} \mathrm{He}$ we have no unambiguous conclusion. Our calculations show that fewbody models of ${ }^{6} \mathrm{He}$ with the correct binding energy predict a reaction cross section on ${ }^{12} \mathrm{C}$ at $800 \mathrm{MeV} /$ nucleon of 705 $\mathrm{mb}$. The discrepancy of this value from the experimental datum of $722 \pm 5$ is as yet unexplained.

For ${ }^{11} \mathrm{Li}$ there are also data at $400 \mathrm{MeV} /$ nucleon and the model is able to reproduce the observed energy dependence without parameter variation, other than the energy dependence of the free $N N$ interaction. We show that the inclusion of the real part of the forward scattering $N N$ amplitude is of little significance in calculated results.

\section{ACKNOWLEDGMENTS}

The financial support of the United Kingdom Engineering and Physical Sciences Research Council (EPSRC) in the form of Grant Nos. GR/J95867 and GR/K33026 is gratefully acknowledged. We would like to thank Dr. Filomena Nunes for providing tabulated two-body wave functions for the

${ }^{11} \mathrm{Be}$ system and Matthew Bush for providing elements of the static density Glauber model code used here.
[1] I. Tanihata et al., Phys. Rev. Lett. 55, 2676 (1985).

[2] I. Tanihata et al., Phys. Lett. 160B, 380 (1985).

[3] I. Tanihata, T. Kobayashi, O. Yamakawa, S. Shimoura, K. Ekuni, K. Sugimoto, N. Takahashi, T. Shimoda, and H. Sato, Phys. Lett. B 206, 592 (1988).

[4] J.S. Al-Khalili and J.A. Tostevin, Phys. Rev. Lett. 76, 3903 (1996).

[5] R.J. Glauber, in Lectures in Theoretical Physics, edited by W.E. Brittin (Interscience, New York, 1959), Vol. 1, p. 315.

[6] W. Czyz and L.C. Maximon, Ann. Phys. (N.Y.) 52, 59 (1969).

[7] P.J. Karol, Phys. Rev. C 11, 1203 (1974).

[8] L.V. Chulkov, B.V. Danilin, V.D. Efros, A.A. Korsheninnikov, and M.V. Zhukov, Europhys. Lett. 8, 245 (1989).

[9] S. Kox et al., Phys. Rev. C 35, 1678 (1987).

[10] K. Yabana, Y. Ogawa, and Y. Suzuki, Phys. Rev. C 45, 2909 (1992); Nucl. Phys. A539, 295 (1992).

[11] J.S. Al-Khalili, I.J. Thompson, and J.A. Tostevin, Nucl. Phys. A581, 331 (1995).

[12] H. Nishioka and R.C. Johnson, Phys. Rev. C 22, 2457 (1980); J. Phys. G 8, 39 (1982).

[13] N. Takigawa, M. Ueda, M. Kuratani, and H. Sagawa, Phys. Lett. B 288, 244 (1992).

[14] M.V. Zhukov, B.V. Danilin, D.V. Federov, J.M. Bang, I.J. Thompson, and J.S. Vaagen, Phys. Rep. 231, 151 (1993).

[15] Y. Ogawa, K. Yabana, and Y. Suzuki, Nucl. Phys. A543, 722 (1992)

[16] T. Kobayashi et al., Phys. Lett. B 232, 51 (1989).

[17] W. Bauhoff, At. Data Nucl. Data Tables 35, 429 (1986).

[18] C.A. Bertulani and H. Sagawa, Nucl. Phys. A588, 667 (1995), and references therein.
[19] V. Franco and G.K. Varma, Phys. Rev. C 18, 349 (1978).

[20] L. Ray, Phys. Rev. C 20, 1857 (1979).

[21] I. Tanihata, J. Phys. G 22, 157 (1996).

[22] M.V. Zhukov, D.V. Fedorov, B.V. Danilin, J.S. Vaagen, J.M. Bang, and I.J. Thompson, Nucl. Phys. A552, 353 (1993).

[23] F.M. Nunes, I.J. Thompson, and R.C. Johnson, Nucl. Phys. A596, 171 (1996).

[24] S.K. Charagi and S.K. Gupta, Phys. Rev. C 41, 1610 (1990).

[25] I.J. Thompson and M.V. Zhukov, Phys. Rev. C 49, 1904 (1994).

[26] J.M. Bang and I.J. Thompson, Phys. Lett. B 270, 201 (1992).

[27] L. Johannsen, A.S. Jensen, and P.G. Hansen, Phys. Lett. B 244, 357 (1990).

[28] I.J. Thompson and M.V. Zhukov, Phys. Rev. C 53, 708 (1996).

[29] I. Tanihata, Nucl. Phys. A488, 113c (1988).

[30] G. Audi and A.H. Wapstra, Nucl. Phys. A565, 66 (1993).

[31] I. Tanihata, D. Hirata, T. Kobayashi, S. Shimoura, K. Sugimoto, and H. Toki, Phys. Lett. B 289, 261 (1992).

[32] S.G. Cooper and R.S. Mackintosh, Phys. Rev. C 43, 1001 (1991); S.G. Cooper, R.S. Mackintosh, A. Csótó, and R.G. Lovas, ibid. 50, 1308 (1994).

[33] J.M. Bang and C. Gignoux, Nucl. Phys. A313, 119 (1979).

[34] S. Sack, L.C. Biedenharn, and G. Breit, Phys. Rev. 93, 321 (1954).

[35] I. Tanihata (private communication).

[36] R. de Tourreil and D.W.L. Sprung, Nucl. Phys. A242, 445 (1975).

[37] R.V. Reid, Ann. Phys. (N.Y.) 50, 411 (1968).

[38] G.E. Brown and A.D. Jackson, The Nucleon-Nucleon Interaction (North-Holland, Amsterdam, 1976). 